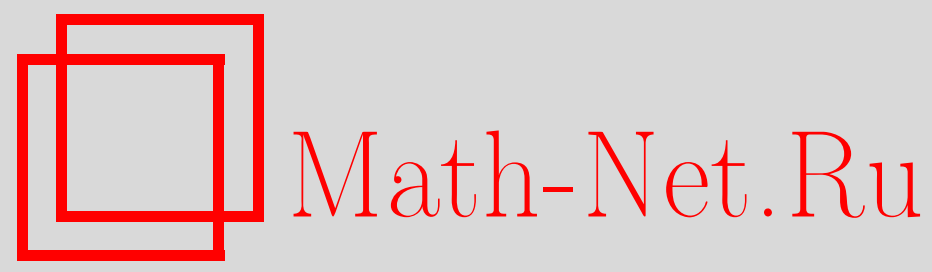

Г. И. Ивченко, М. В. Солдаткина, Статистические задачи для случайных подстановок с цензурированными данными, Дискрет. матем., 2012, том 24, выпуск 4, 104-113

DOI: https://doi.org/10.4213/dm1214

Использование Общероссийского математического портала Math-Net.Ru подразумевает, что вы прочитали и согласны с пользовательским соглашением http://www . mathnet.ru/rus/agreement

Параметры загрузки:

IP : 18.209 .158 .208

26 апреля 2023 г., 10:26:40 
УДК 519.2

\section{Статистические задачи для случайных подстановок с цензурированными данными}

() 2012 г. Г. И. Ивченко, М. В. Солдаткина

В $d$-мерной параметрической модели случайных подстановок степени $n$ решаются задачи асимптотического при $n \rightarrow \infty$ оценивания неизвестных параметров и проверки различных гипотез о них по неполным данным, когда в подстановке наблюдаются лишь циклы некоторой ограниченной длины. Также рассматривается случай больших выборок и строится критерий однородности для него.

\section{1. Введение}

В [2] была введена следующая $d$-параметрическая модель случайных $n$-подстановок, то есть взаимно однозначных отображений множества $X_{n}=\{1,2, \ldots, n\}$ в себя.

Пусть задано некоторое разбиение множества $X_{n}$ :

$$
X_{n}=\bigcup_{j=1}^{d} A_{j}, \quad A_{i} \cap A_{j}=\varnothing, \quad i \neq j,
$$

и пусть $\mathbf{c}(n)=\left(c_{1}, c_{2}, \ldots, c_{n}\right)$ есть цикловая структура $n$-подстановки $s$, именно, $c_{i}$ есть число циклов длины $i, i=1, \ldots, n$, причем

$$
\sum_{i} i c_{i}=n
$$

Циклы подстановки $s$, длины которых являются элементами подмножества $A_{j}$, называются $A_{j}$-циклами, их число обозначается

$$
C_{A_{j}}(n)=\sum_{i \in A_{j}} c_{i}, \quad j=1, \ldots, d .
$$

Если подмножество $A_{j}$ имеет вид

$$
A_{j}=\{k: k=l d+j, l \geqslant 0\}
$$

для некоторых целых $d \geqslant 2$ и $1 \leqslant j \leqslant d$, то говорят о конгруэнтных циклах (см. [1], c. 187). 
Далее, на множестве всех $n$-подстановок $S_{n}=\{s\}$ задается параметрическая вероятностная мера вида

$$
\mathbf{P}_{\theta}(s)=I\left(\sum_{i=1}^{n} i c_{i}=n\right) \frac{1}{H_{n}(\theta)} \prod_{j=1}^{d} \theta_{j}^{C_{A_{j}}(n)},
$$

где $I(\cdot)$ - индикатор, $\theta=\left(\theta_{1}, \ldots, \theta_{d}\right), \theta_{j} \geqslant 0, j=1, \ldots, d,-$ произвольные параметры, не равные нулю одновременно, и $H_{n}(\theta)$ - необходимый нормирующий множитель, имеющий вид

$$
H_{n}(\theta)=n !\left[z^{n}\right] \exp \left\{\sum_{j=1}^{d} \theta_{j} \sum_{i \in A_{j}} \frac{z^{i}}{i}\right\}
$$

здесь и далее

$$
\left[z^{n}\right] f(z)=\operatorname{coef}_{z^{n}} f(z) .
$$

В [2] доказана асимптотическая, при $n \rightarrow \infty$, нормальность вектора

$$
\mathbf{C}(n)=\left(C_{A_{1}}(n), \ldots, C_{A_{d}}(n)\right)
$$

чисел конгруэнтных циклов случайной подстановки в такой модели и решены соответствующие задачи проверки статистических гипотез о параметре $\theta=\left(\theta_{1}, \ldots, \theta_{d}\right)$.

Подчеркнем, что в этих исследованиях предполагается известной вся цикловая последовательность $\mathbf{c}(n)=\left(c_{1}, c_{2}, \ldots, c_{n}\right)$, то есть имеется полная информация о наблюдаемой подстановке, и соответствующие выводы имеют асимптотический (при $n \rightarrow \infty$ ) характер. Но в этом случае не всегда является реалистичным предположение о том, что мы можем наблюдать всю цикловую последовательность с $(n)$. Может быть и так, что наблюдению доступно лишь какое-то ограниченное число $k$ ее первых членов $c_{1}, c_{2}, \ldots, c_{k}$, в этом случае говорят о цензурированных (неполных) данных. Статистические задачи для случайных подстановок с неполными данными в рамках однопараметрической модели Эванса, когда подстановка $s \in S_{n}$ наблюдается с вероятностью, пропорциональной $\theta^{c(n)}$, где

$$
c(n)=c_{1}+c_{2}+\ldots+c_{n}
$$

есть общее число циклов подстановки $s$, рассматривались в [4]. В настоящей работе аналогичный подход применяется к описанной выше $d$-параметрической модели с конгруэнтными циклами.

Именно, будем предполагать, что в наблюдаемой подстановке $s \in S_{n}$ для каждого $j=1, \ldots, d$ доступно подсчету лишь число $A_{j}$-циклов с длинами, не превосходящими заданного уровня $K_{j}$. В таком случае, пусть

$$
\xi_{j}(n)=\sum_{i \in A_{j}} c_{i} I\left(i \leqslant K_{j}\right), \quad j=1, \ldots, d,
$$

есть наши исходные данные (количества наблюдаемых $A_{j}$-циклов). Рассмотрим различные вопросы статистического вывода для модели (1)-(4) именно по таким неполным данным (5). При этом будем предполагать, что порядок подстановки $n \rightarrow \infty$, а параметры цензурирования $K_{j}, j=1, \ldots, d$, фиксированы.

Основой для дальнейших выводов будет служить следующее утверждение об асимптотическом распределении наблюдаемых статистик $c_{1}, c_{2}, \ldots$, то есть начальных членов цикловой структуры подстановки. 
Теорема 1. Для случайной подстановки s в модели (1)-(4) при $n \rightarrow \infty$ числа цииклов ограниченной длины асимптотически независимы, и при этом число $A_{j}$-циклов длины $i$ имеет в пределе распределение Пуассона П $\left(\theta_{j} / i\right)$.

Доказательство этой теоремы приведено в приложении.

Следствие 1. Наблюдаемье статистики (5) асимптотически независимы, и

$$
\mathscr{L}\left(\xi_{j}(n)\right) \rightarrow \Pi\left(\theta_{j} \lambda_{j}\right), \quad \lambda_{j}=\sum_{i \in A_{j}} \frac{1}{i} I\left(i \leqslant K_{j}\right)=\sum_{l \leqslant\left(K_{j}-j\right) / d} \frac{1}{l d+j}
$$

Из этих результатов следует, во-первых, что статистические выводы о каждом из параметров $\theta_{j}$ можно делать независимо по наблюдению лишь соответствующей статистики $\xi_{j}(n)$, и, во-вторых, исходная проблема в асимптотике сводится к соответствующим статистическим задачам для пуассоновской модели с неизвестным параметром, решение которых достаточно хорошо известно. Используя известные результаты для пуассоновской модели, мы в разделе 2 приводим решение задач точечного и доверительного оценивания параметров $\theta_{j}$ нашей модели, и в разделе 3 - задач проверки статистических гипотез. В разделе 4 анализируется многовыборочный случай (наблюдается большое число независимых подстановок), и в разделе 5 решается задача проверки гипотезы однородности для этой ситуации.

\section{2. Оценивание параметров}

Пусть имеется $N$ независимых подстановок $s_{1}, \ldots, s_{N}$, полученных при одном и том же значении неизвестного параметра $\theta=\left(\theta_{1}, \ldots, \theta_{d}\right)$, и, тем самым, имеется выборка (набор наблюдаемых статистик (5))

$$
\left(\xi_{1}{ }^{(k)}(n), \ldots, \xi_{d}^{(k)}(n)\right), \quad k=1, \ldots, N .
$$

Как отмечено во введении, компоненты этих векторов асимптотически, при $n \rightarrow \infty$ и фиксированных уровнях цензурирования $K_{j}$, независимы и распределены в соответствии с (6), поэтому для суммарного числа наблюдаемых $A_{j}$-циклов

$$
T_{j}=T_{j}(N, n)=\sum_{k=1}^{N} \xi_{j}^{(k)}(n)
$$

будет выполняться предельное соотношение

$$
\mathscr{L}\left(T_{j}(N, n)\right) \rightarrow \Pi\left(N \theta_{j} \lambda_{j}\right)
$$

Это соотношение сводит задачу оценивания параметров $\theta_{j}$ модели (1)-(4) к задаче оценивания неизвестного параметра пуассоновской модели.

Известно (см., например, [6, §3.4]), что оптимальной, то есть несмещенной с минимальной дисперсией, оценкой сходящегося при всех $\theta>0$ степенного ряда

$$
\tau(\theta)=\sum_{i \geqslant 0} a_{i} \theta^{i}
$$


по наблюдению над случайной величиной $X$ с пуассоновским распределением П $(\theta)$ является статистика

$$
\tau^{*}=\sum_{i \geqslant 0} a_{i}(X)_{i}
$$

где

$$
(X)_{i}=X(X-1) \cdots(X-i+1), \quad i \geqslant 1, \quad(X)_{0}=1 .
$$

Учитывая это и соотношение (9), мы можем сформулировать следующее общее утверждение для нашей модели.

Предложение 1. Асимптотически оптимальной оценкой сходящฺегося при всех $\theta_{j}>0$ степенного ряда

$$
\tau\left(\theta_{j}\right)=\sum_{i \geqslant 0} a_{i} \theta_{j}^{i}
$$

является статистика

$$
\tau^{*}=\sum_{i \geqslant 0} \frac{a_{i}\left(T_{j}(N, n)\right)_{i}}{\left(N \lambda_{j}\right)^{i}}
$$

в частности, асимптотически оптимальная оценка параметра $\theta_{j}$ имеет вид

$$
\theta_{j}^{*}=\frac{T_{j}(N, n)}{N \lambda_{j}} .
$$

Соответствуюший же $\gamma$-доверительный интервал для $\theta_{j}$ асимптотически имеет вид

$$
\left(\frac{1}{2 N \lambda_{j}} \chi_{(1-\gamma) / 2,2 T_{j}}^{2}, \frac{1}{2 N \lambda_{j}} \chi_{(1+\gamma) / 2,2 T_{j}+2}^{2}\right),
$$

где $\chi_{p, r}^{2}$ есть р-квантиль распределения хи-квадрат с r степенями свободы.

\section{3. Проверка гипотез}

Если требуется проверить какие-то гипотезы о частных значениях параметров $\theta_{j}$ в обсуждаемой ситуации, то на основании соотношения (9) надо воспользоваться общей теорией для пуассоновской модели, учитывая при этом специфику тестовых статистик $T_{j}(N, n)$, с тем лишь замечанием, что соответствующие алгоритмы в нашем случае будут иметь характер асимптотических утверждений. Продемонстрируем это на конкретном примере проверки простой гипотезы $H_{0}: \theta_{j}=\theta_{j 0}$ против правосторонней альтернативы $H_{1}^{+}$: $\theta_{j}>\theta_{j 0}$. Известно (см. [6], §5.3), что в задачах такого типа существует равномерно наиболее мощный (РНМ) критерий, который строится следующим образом. Обозначим в нашем случае

$$
\theta_{0}=\theta_{j 0} N \lambda_{j}
$$

и при заданной вероятности ошибки первого рода $\alpha$ определим целое число $t_{\alpha}$ условием

$$
\alpha^{\prime \prime} \equiv \sum_{m=t_{\alpha}+1}^{\infty} \frac{\theta_{0}^{m}}{m !} e^{-\theta_{0}}<\alpha \leqslant \sum_{m=t_{\alpha}}^{\infty} \frac{\theta_{0}^{m}}{m !} e^{-\theta_{0}} \equiv \alpha^{\prime} .
$$


Если здесь $\alpha=\alpha^{\prime}$, то искомый критерий является нерандомизированным и асимптотически задается критической областью

$$
\mathscr{X}_{\alpha}^{+}=\left\{T_{j}(N, n) \geqslant t_{\alpha}\right\} .
$$

Если же в (12) имеет место строгое неравенство $\alpha<\alpha^{\prime}$, то критерий является рандомизированным и задается критической функцией

$$
\Phi_{\alpha}\left(T_{j}\right)= \begin{cases}1, & T_{j}>t_{\alpha}, \\ \frac{\alpha-\alpha^{\prime \prime}}{\alpha^{\prime}-\alpha^{\prime \prime}}, & T_{j}=t_{\alpha}, \\ 0, & T_{j}<t_{\alpha}\end{cases}
$$

В любом случае мощность этого критерия при произвольной альтернативе

$$
\theta_{1}=\theta_{j} N \lambda_{j}>\theta_{0}
$$

вычисляется по формуле

$$
W\left(\theta_{1}\right) \sim \sum_{m=t_{\alpha}+1}^{\infty} \frac{\theta_{1}^{m}}{m !} e^{-\theta_{1}}+\left(\alpha-\alpha^{\prime \prime}\right)\left(\frac{\theta_{1}}{\theta_{0}}\right)^{t_{\alpha}} e^{\theta_{0}-\theta_{1}} .
$$

Замечание 1. В случае $\alpha<\alpha^{\prime}$, вместо рандомизированного критерия можно также использовать нерандомизированные РНМ критерии

$$
\mathscr{X}_{\alpha^{\prime}}^{+}=\left\{T_{j} \geqslant t_{\alpha}\right\}
$$

или

$$
\mathscr{2}_{\alpha^{\prime \prime}}^{+}=\left\{T_{j} \geqslant t_{\alpha}+1\right\}
$$

с уровнями значимости, асимптотически равными соответственно $\alpha^{\prime}$ и $\alpha^{\prime \prime}$.

Аналогично анализируется задача $\left(H_{0}, H_{1}^{-}\right)$с левосторонней альтернативой, а при двусторонней альтернативе критерий задается объединением двух односторонних критических областей, то есть имеет вид

$$
\mathscr{X}_{\alpha}=\left\{T_{j} \geqslant t_{\alpha_{1}}\right\} \cup\left\{T_{j} \leqslant t_{\alpha_{2}}\right\}, \quad \alpha_{1}+\alpha_{2}=\alpha .
$$

Замечание 2. Случай $\theta_{j 0}=1, j=1, \ldots, d$, соответствует наиболее важной для приложений гипотезе о равновероятности подстановок, и изложенная методика дает новые критерии проверки этой гипотезы, учитывающие широкий класс специальных альтернатив, при которых $\theta_{j} \neq 1$ для некоторых $j$.

\section{4. Большие выборки}

Если число $N$ наблюдаемых подстановок велико, то можно применить теорию больших выборок (при $N \rightarrow \infty$ ) и получить более сильные выводы. В этом случае из (8)-(9) следует, что для нормированной статистики

$$
\widetilde{T}_{j}=\widetilde{T}_{j}(N, n)=\frac{T_{j}(N, n)}{N \lambda_{j}}
$$


справедлива нормальная аппроксимация

$$
\mathscr{L}\left(\widetilde{T}_{j}\right) \sim \mathcal{N}\left(\theta_{j}, \frac{\theta_{j}}{N \lambda_{j}}\right),
$$

и, тем самым, статистические выводы о параметрах $\theta_{j}$ можно получать, используя соответствующую теорию нормальной модели [4]. Аналогичная ситуация рассматривалась в [3], поэтому, следуя ей, можно сформулировать для нашего случая следующие утверждения.

Предложение 2. Статистика $\widetilde{T}_{j}$ является асимптотически несмещенной и асимптотически эффективной оценкой параметра $\theta_{j}$; такими же свойствами обладает статистика $\tau\left(\widetilde{T}_{j}\right)$ как оценка $\tau\left(\theta_{j}\right)$ для любой дифференцируемой функции $\tau$.

Предложение 3. Асимптотическим $\gamma$-доверительным интервалом для параметрической функции $\tau\left(\theta_{j}\right)$ с непрерывной производной $\tau^{\prime}\left(\theta_{j}\right)$ является интервал

$$
\left(\tau\left(\widetilde{T}_{j}\right) \mp z_{\gamma} \tau^{\prime}\left(\widetilde{T}_{j}\right) \sqrt{\frac{\widetilde{T}_{j}}{N \lambda_{j}}}\right),
$$

где

$$
z_{\gamma}=\Phi^{-1}\left(\frac{1+\gamma}{2}\right)
$$

и $\Phi^{-1}(t)$ - обратная функция к стандартной нормальной функции распределения $\Phi(x)$.

Предложение 4. Критерий уровня значимости $\alpha$ для проверки гипотезы $H_{0}: \theta_{j}=1$ при левосторонней альтернативе $H_{1}^{-}: \theta_{j}<1$ асимптотически задается критической областью

$$
\mathscr{X}_{\alpha}^{-}(N)=\left\{\tilde{T}_{j}<1-u_{\alpha} / \sqrt{N \lambda_{j}}\right\}, \quad u_{\alpha}=\Phi^{-1}(1-\alpha)
$$

пороговыми здесь являются альтернативы вида

$$
H_{1 N}^{-}: \theta_{j}=\theta_{j}(N)=1-\frac{t}{\sqrt{N \lambda_{j}}}, \quad t>0,
$$

и мощьность критерия (18) при таких близких альтернативах удовлетворяет при $N \rightarrow \infty$ соотношению

$$
W_{N}\left(\theta_{j}\right)=\mathbf{P}\left(\mathscr{X}_{\alpha}^{-}(N)\right) \rightarrow \Phi\left(t-u_{\alpha}\right) .
$$

Аналогично, в задаче $\left(H_{0}, H_{1}^{+}: \theta_{j}>1\right)$ критерий уровня значимости $\alpha$ асимптотически имеет вид

$$
\mathscr{X}_{\alpha}^{+}(N)=\left\{\widetilde{T}_{j}>1+u_{\alpha} / \sqrt{N \lambda_{j}}\right\}
$$

и его мощность при близких альтернативах

$$
H_{1 N}^{+}: \theta_{j}=\theta_{j}(N)=1+\frac{t}{\sqrt{N \lambda_{j}}}, \quad t>0,
$$


асимптотически равна $\Phi\left(t-u_{\alpha}\right)$.

Наконеи, в задаче с двусторонней альтернативой $\left(H_{0}, H_{1}=H_{1}^{-} \cup H_{1}^{+}\right)$критерий асимптотически имеет вид

$$
\mathscr{X}_{\alpha}(N)=\left\{\left|\widetilde{T}_{j}-1\right|>\frac{u_{\alpha / 2}}{\sqrt{N \lambda_{j}}}\right\},
$$

и его мощность при близких альтернативах

$$
H_{1 N}: \theta_{j}=\theta_{j}(N)=1+\frac{t}{\sqrt{N \lambda_{j}}}, \quad t \neq 0,
$$

асимптотически равна $\Phi\left(t-u_{\alpha / 2}\right)+\Phi\left(-t-u_{\alpha / 2}\right)$.

Сформулированные в предложении 4 критерии позволяют контролировать значения отдельных координат параметрического вектора $\theta=\left(\theta_{1}, \ldots, \theta_{d}\right)$. Вместе с тем, желательно уметь также проверять гипотезы и о полном этом векторе. Важнейшей такой гипотезой является утверждение

$$
H_{0}: \theta_{1}=\theta_{2}=\ldots=\theta_{d}=1,
$$

означающее, что подстановки выбираются равновероятно из $S_{n}$.

В случае, если эта гипотеза справедлива, то из (15)-(16) следует, что статистики

$$
T_{j}^{*}(N)=\frac{T_{j}-N \lambda_{j}}{\sqrt{N \lambda_{j}}}, \quad j=1, \ldots, d,
$$

асимптотически распределены по стандартному нормальному закону и асимптотически независимы. Следовательно, сумма их квадратов асимптотически распределена по закону $\chi^{2}(d)$ :

$$
\mathscr{L}\left(T^{2}(N) \equiv \sum_{j=1}^{d} \frac{\left(T_{j}-N \lambda_{j}\right)^{2}}{N \lambda_{j}}\right) \rightarrow \chi^{2}(d) .
$$

Этот результат позволяет сформулировать следующий критерий согласия для гипотезы равновероятности $H_{0}$, учитывающий всю исходную информацию.

Предложение 5. При заданном уровне значимости $\alpha$,

$$
H_{0} \text { отвергается } \Longleftrightarrow\left\{T^{2}(N)>\chi_{1-\alpha, d}^{2}\right\} .
$$

\section{5. Гипотеза однородности}

Выше предполагалось, что в процессе наблюдения над подстановками $s_{1}, \ldots, s_{N}$ параметр $\theta=\left(\theta_{1}, \ldots, \theta_{d}\right)$ модели (1)-(4) остается неизменным, что на самом деле является гипотезой, которая сама должна быть подвергнута статистической проверке; такая гипотеза и называется гипотезой однородности. Здесь предлагается асимптотический (для больших выборок) вариант соответствующего критерия однородности.

Итак, теперь считаем, что каждый из векторов (7) получен, вообще говоря, при своем (но неизвестном) значении параметра $\theta$. Обозначим значение этого параметра для подстановки $s_{k}$ через $\theta^{(k)}=\left(\theta_{1}^{(k)}, \ldots, \theta_{d}^{(k)}\right)$. Тогда гипотеза однородности есть утверждение

$$
H_{0}: \theta^{(1)}=\ldots=\theta^{(N)} \text {. }
$$


В силу предположения о независимости подстановок и соотношения (6), можно считать, что при гипотезе $H_{0}$

$$
\left(\xi_{j}^{(k)}(n), k=1, \ldots, N\right)=\left(X_{1 j}, \ldots, X_{N j}\right)
$$

представляют собой независимую выборку из распределения Пуассона П $\left(\theta_{j} \lambda_{j}\right)$ при некотором $\theta_{j}>0$; далее для краткости полагаем

$$
\alpha_{j}=\theta_{j} \lambda_{j}
$$

при этом, в силу следствия 1 из введения, для разных j выборки (22) асимптотически независимы.

Построим выборочные среднее и дисперсию выборки (22):

$$
\begin{aligned}
\bar{X}_{j} & =\frac{1}{N} \sum_{i=1}^{N} X_{i j}, \\
S_{j}^{2} & =\frac{1}{N} \sum_{i=1}^{N}\left(X_{i j}-\bar{X}_{j}\right)^{2},
\end{aligned}
$$

и введем статистику

$$
T_{N j}=\frac{1}{\bar{X}_{j}} \sqrt{\frac{N-1}{2}}\left(\bar{X}_{j}-\frac{N}{N-1} S_{j}^{2}\right) .
$$

Как показано в [4], эта статистика при гипотезе $H_{0}$ распределена асимптотически по стандартному нормальному закону $\mathcal{N}(0,1)$, а так как для разных $j$ такие статистики асимптотически независимы, то объединенная статистика

$$
T_{N}^{2}=\sum_{j=1}^{d} T_{N j}^{2}
$$

при гипотезе $H_{0}$ будет асимптотически иметь распределение $\chi^{2}(d)$.

Тем самым, основываясь на статистике $T_{N}^{2}$, можно предложить следующий критерий согласия для гипотезы $H_{0}$.

Предложение 6. При заданном уровне значимости $\alpha$, гипотеза однородности $\mathrm{H}_{0}$ отвергается тогда и только тогда, когда

$$
T_{N}^{2} \geqslant \chi_{1-\alpha, d}^{2}
$$

\section{Приложение}

В теории случайных $n$-подстановок хорошо известен факт асимптотической, при $n \rightarrow \infty$, независимости и асимптотической пуассоновости начальных членов цикловой последовательности $\mathbf{c}(n)=\left(c_{1}, c_{2}, \ldots, c_{n}\right)$ случайной равновероятной подстановки, при этом

$$
\mathscr{L}\left(c_{i}\right) \rightarrow \Pi\left(\frac{1}{i}\right)
$$


для любого конечного $i$.

Аналогичное свойство имеет место и для модели Эванса, причем в этом случае

$$
\mathscr{L}\left(c_{i}\right) \rightarrow \Pi\left(\frac{\theta}{i}\right) .
$$

Это свойство асимптотической независимости и пуассоновости чисел циклов конечной длины сохраняется и для общей параметрической модели, введенной в [5], согласно которой произвольная подстановка $s \in S_{n}$ наблюдается с вероятностью, пропорциональной $\prod_{i} \theta_{i}^{c_{i}}$, где $\theta=\left(\theta_{1}, \ldots, \theta_{n}\right), \theta_{\bullet} \geqslant 0$ - параметр меры. В этом случае для производящей функции цикловой структуры с $(n)$ справедливо представление

$$
\mathbf{E}_{\theta} \prod_{i=1}^{n} t_{i}^{c_{i}}=\frac{H_{n}(t \theta)}{H_{n}(\theta)}
$$

где

$$
H_{n}(\theta)=n !\left[z^{n}\right] \exp \{a(z ; \theta)\}
$$

и

$$
a(z ; \theta)=\sum_{i=1}^{n} \theta_{i} \frac{z^{i}}{i}
$$

Отсюда следует, что производящая функция для произвольного конечного числа $k$ начальных членов $c_{1}, c_{2}, \ldots, c_{k}$ имеет вид

$$
\mathbf{E}_{\theta} \prod_{i=1}^{k} t_{i}^{c_{i}}=\frac{n !}{H_{n}(\theta)}\left[z^{n}\right] \exp \left\{a(z ; \theta)+\sum_{i=1}^{k} \theta_{i}\left(t_{i}-1\right) \frac{z^{i}}{i}\right\} .
$$

Далее, используя стандартную технику метода перевала, нетрудно получить, что при $n \rightarrow \infty$ и фиксированных $\theta$ • справедливо соотношение

$$
\mathbf{E}_{\theta} \prod_{i=1}^{k} t_{i}^{c_{i}} \rightarrow \exp \left\{\sum_{i=1}^{k} \frac{\theta_{i}}{i}\left(t_{i}-1\right)\right\},
$$

которое означает асимптотическую независимость величин $c_{1}, \ldots, c_{k}$ и их пуассоновскую сходимость:

$$
\mathscr{L}\left(c_{i}\right) \rightarrow \Pi\left(\frac{\theta_{i}}{i}\right)
$$

Утверждение теоремы, сформулированной во введении, является следствием этого результата, поскольку для всех $A_{j}$-циклов параметры $\theta$ в в рассматриваемой модели одинаковы.

\section{Список литературы}

1. Сачков В. Н., Введение в комбинаторные методы дискретной математики. МЦНМО, Москва, 2004. 
2. Соболева М. В., Асимптотическая нормальность чисел конгруэнтных циклов в случайных подстановках. Дискретная математика (2012) 24, №1, 123-131.

3. Ивченко Г. И., Медведев Ю. И., Статистика параметрической модели случайных подстановок. Труды по дискретной математике (2004) 8, 116-127.

4. Ивченко Г. И., Медведев Ю. И., Статистические выводы для случайных подстановок по неполным данным. Труды по дискретной математике (2006) 9, 66-76.

5. Ивченко Г. И., Медведев Ю. И., Случайные подстановки: общая параметрическая модель. Дискретная математика (2006) 18, №4, 105-112.

6. Ивченко Г. И., Медведев Ю. И., Введение в математическую статистику. ЛКИ/URSS, Москва, 2010.

7. Ивченко Г. И., Медведев Ю. И., О случайных подстановках. Труды по дискретной математике (2002) 5, 73-92.

Статья поступила 28.08.2012. 